\title{
Nathan Rosenberg: historiador das revoluções tecnológicas e de suas interpretações econômicas*
}

\author{
Eduardo da Motta e Albuquerque*
}

Recebido: 07/10/2016 Versão Revisada (entregue): 11/12/2016 Aprovado: 11/01/2017

\section{RESUMO}

Nathan Rosenberg (1927-2015) é um dos pilares da elaboração neoschumpeteriana e, ao lado de Christopher Freeman e Richard Nelson, um dos responsáveis pelo conjunto de obras que estabelecem pontos de partida para a abordagem evolucionista em meados dos anos 1970. Este artigo discute um elemento paradoxal na obra de Rosenberg: um autor cético quanto às ondas longas do desenvolvimento capitalista que gerou trabalhos decisivos para entender o impacto de longo prazo de revoluções tecnológicas. Parte de Schumpeter e do esquema invenção-inovaçãoimitação para demonstrar como o processo é mais complexo e multifacetado. O resultado é uma visão bem mais elaborada da propagação de revoluçôes tecnológicas, sistematizada no estudo da trajetória de diversas inovações radicais: motor a combustão, química, eletricidade e eletrônica. Essa contribuição teórica alimenta um diálogo com a elaboração das general purpose technologies (GPTs), que constituem uma abordagem que contém um diálogo com formulaçóes neoschumpeterianas sobre as ondas longas do desenvolvimento capitalista.

PALAVRAS-CHAVE | Inovação Tecnológica; História da Tecnologia; Tecnologias de Propósito Geral

Códigos-JEL | B25; O31; O33

* O autor agradece o apoio do CNPq (Processo 302857/2015-0). Durante a elaboração deste artigo, conversas com Richard Nelson, Roberto Mazzoleni, Leonardo Costa Ribeiro e Wilson Suzigan, além dos comentários críticos de Marcelo Pinho e Renato Garcia, enriqueceram tanto a sua redação como sua revisão. Os erros são responsabilidade do autor.

** Centro de Desenvolvimento e Planejamento Regional (Cedeplar), Universidade Federal de Minas Gerais (UFMG), Belo Horizonte (MG), Brasil.E-mail: albuquer@cedeplar.ufmg.br. 


\title{
Nathan Rosenberg: historian of technological revolutions and their economic interpretations
}

\begin{abstract}
Nathan Rosenberg (1927-2015) and his works, together with Christopher Freeman and Richard Nelson, are starting points for evolutionary approach in the mid-1970s. This article discusses a paradox in Rosenberg's work: on the one hand, he is an author skeptical on the elaboration on long waves of capitalist development, on the other hand his work is key to understand the long-term impact of technological revolutions. His critical interpretation of Schumpeter's sequential scheme (invention-innovation-imitation) unveils how the process is more complex and multifaceted. The result is a much more elaborated view of the spread of technological revolutions, based upon a systematic study of the trajectory of several radical innovations: combustion engine, chemistry, electricity and electronics. Rosenberg's contributions are incorporated in the emerging concept of General Purpose Technologies (GPTs) - an approach that has a dialogue with neoschumpeterian formulations on long waves of capitalist development.
\end{abstract}

KEYWORDS | Technological Innovation; History of Technology; General Purpose Technologies

JEL CODES | B25; O31; O33 


\section{Introdução}

Nathan Rosenberg (1927-2015), professor emérito da Universidade de Stanford, onde trabalhou entre 1974 e 2002, é um dos pilares da elaboração neoschumpeteriana $^{1}$ e um dos responsáveis pelas obras que estabelecem pontos de partida para a abordagem evolucionista em meados dos anos 1970: Freeman (1974), Rosenberg (1976) e Nelson e Winter (1977).

Historiador da economia e do pensamento econômico voltado para a temática do progresso tecnológico, Rosenberg usou esse instrumental para analisar trajetórias tecnológicas de inovações radicais e outras questôes decisivas sobre a dinâmica tecnológica em geral.

Para apresentar um aspecto de uma obra vasta e influente, este artigo discute um elemento paradoxal: embora Rosenberg tenha demonstrado seu ceticismo quanto a um tópico essencial da elaboração de Schumpeter - o papel das ondas longas do desenvolvimento capitalista -, sua obra gerou trabalhos decisivos para entender o impacto de longo prazo de revoluções tecnológicas - uma contribuição inestimável ao estudo dos movimentos de longo prazo investigados pelas pesquisas na linha aberta por Kondratiev.

Não é simples lidar com esse elemento paradoxal - as contribuiçõos de um crítico da elaboração das ondas longas para o desenvolvimento dessa abordagem dada a fertilidade do autor e a vastidão da obra legada por Rosenberg. Este artigo opta por combinar, por um lado, uma exposição que segue uma sequência cronológica e, por outro, um percurso que tem como fio condutor o aparecimento e o desenvolvimento de questôes relacionadas às revoluções tecnológicas.

Para tratar desse paradoxo, o artigo está estruturado em sete seçōes, incluindo esta introdução. A segunda seção apresenta o papel fundamental de Rosenberg na emergência da elaboração evolucionária. Na terceira, discute-se como Rosenberg, um leitor e crítico de Schumpeter, adota o esquema simples sugerido pelo autor de Business cycles (invenção-inovação-imitação) como ponto de partida para demonstrar a maior complexidade desses processos. A quarta seção sistematiza o ceticismo de Rosenberg em relação às ondas longas do desenvolvimento capitalista e a quinta traz um balanço intermediário da elaboração de Rosenberg em meados dos anos 1990 - um período em que o papel das instituições e da mudança institucional ganha 
atenção em sua produção. Nesse ponto está madura uma visão elaborada sobre a origem e a propagação de revoluções tecnológicas. A sexta seção discute como essa elaboração alimenta, a partir do final da década de 1990, um diálogo em torno das general purpose technologies (GPTs). Por fim, a obra de Rosenberg é avaliada como uma contribuição permanente para a compreensão da dinâmica de longo prazo da economia capitalista: um historiador das revoluções tecnológicas, cético sobre as ondas longas que Kondratiev e Schumpeter pensaram como sua expressão, contribuindo para retomar a discussão sobre impactos de longo prazo de inovações tecnológicas importantes em um novo patamar, a partir da crescente influência do conceito das GPTs. Nessa seção final, são explorados temas de uma agenda de pesquisa gerada por essas contribuições de Rosenberg.

\section{Rosenberg e a emergência da abordagem evolucionária}

A contribuição teórica específica de Rosenberg para a emergência da elaboração evolucionária é derivada da sua condição de historiador - um ativo pesquisador da história da economia e do pensamento econômico, com foco especial em questóes relacionadas ao progresso tecnológico. Rosenberg usou esse instrumental para investigar a dinâmica tecnológica em geral, por meio de uma ferramenta metodológica: "historical reconnaissance mission" (ROSENBERG, 1969, p. 108). ${ }^{2}$

Perspectives on technology apresenta a contribuição inicial de Rosenberg (1976) para a abordagem evolucionária, sintetizando investigações por ele realizadas até meados dos anos 1970. Trata-se de uma coletânea de ensaios previamente publicados por Rosenberg entre 1963 e 1975, o que sugere uma influência dispersa, mas importante, na produção de temas decisivos para a origem da elaboração evolucionária.

A primeira parte desse livro intitula-se "Some origins of American technology", um título que tem uma identidade notável com o subtítulo de um livro de 2000 ("Some American perspectives") - uma indicação de que Rosenberg nunca abandonou a posição de investigador das particularidades históricas do desenvolvimento econômico e tecnológico dos Estados Unidos.

O capítulo que abre a primeira parte de Perspectives on technology foi publicado originalmente em 1963: "Technological change in the machine tool industry, 1840-1910”. E É um artigo clássico para definir o papel da indústria de bens de

2 Posteriormente, Rosenberg (1982, p. 142) afirmou que esse tipo de empreendimento intelectual - "preliminary reconnaissance" - seria apenas "the first small step on a long intellectual journey".

3 Para o argumento deste texto, esse capítulo de Rosenberg é crucial, pois no capítulo 5 de Schumpeter and the endogeneity of technology, como será mencionado adiante, há uma referência a este trabalho como relacionado à elaboração das GPTs. 
capital no desenvolvimento econômico, que descreve o crescimento dessa indústria nos Estados Unidos. Por um lado, Rosenberg discute o conceito de desintegração vertical e a subsequente maior especialização existente em um mercado que cresce - o surgimento de empresas especializadas na produção de máquinas-ferramentas -, em um diálogo com G. Stigler. Por outro lado, ao sugerir que esse conceito é insuficiente para captar a importância histórica e dinâmica do setor, o autor apresenta o conceito de convergência tecnológica para descrever a importância do impacto desse setor sobre uma multiplicidade de outras indústrias em desenvolvimento nos Estados Unidos de então - armas, máquinas de costura, bicicletas e automóveis -, com ênfase no papel de sequências históricas - "doing things successfully creates a capacity for doing other things” (ROSENBERG, 1976, p. 30). Nesse artigo está uma discussão pioneira sobre a complementariedade entre tecnologias (ROSENBERG, 1976, p. 11). ${ }^{4}$

Essa linha de estudos com forte base histórica compõe-se de dois outros trabalhos anteriores ao Perspectives on technology. Em 1969, Rosenberg organizou uma edição de um documento encomendado no Reino Unido após a exposição industrial do Crystal Palace de 1851, importante para a compreensão da transição da liderança tecnológica do Reino Unido para os Estados Unidos: o "Report of the commitee on the machinery of the United States of America. Presented to the House of Commons, in pursuance of their address of the 10th July 1855". Na introdução dessa edição, Rosenberg (1969, p. 1) destaca a importância histórica dos documentos, pois "[t]he reports also simbolize, in an important sense, the emergence of the United States as a world economic power. Indeed, they were instrumental in initiating a sizeable reverse flow of technical knowledge which had for so long flowed from the Old World to the New". A introdução de Rosenberg é um cuidadoso estudo sobre o que os britânicos nomearam como "American System of Manufacturing" - o estado da tecnologia nos Estados Unidos na primeira metade da década de 1850. Posteriormente, em 1972, Rosenberg publicou o livro Technology and American economic growth, que descreve a transição ao longo do século XIX dos Estados Unidos de um imitador (capítulo 3) para um iniciador (capítulo 4) de tecnologias. Certamente é um estudo que contribui para a percepção de Rosenberg sobre a complexidade envolvida na atividade de imitação.

Esses estudos históricos combinam-se com incursões de Rosenberg na área do pensamento econômico - The emergence of economic ideas foi lançado em 1994, mas

4 Dosi (1982, p. 154), ao desenvolver o conceito de paradigmas e trajetórias tecnológicas, refere-se a Rosenberg (1976) quando trata de complementariedades entre trajetórias. 
dos 12 capítulos desse livro, nove já haviam sido publicados até 1976, nos quais Rosenberg discute as formulações de Mandeville (capítulo 1), Adam Smith (capítulos 2 a 7) e Marx (capítulos 9 e 10). Os outros três capítulos produzidos na década de 1990 tratam de Babbage, Schumpeter e Stigler (este como "Adam Smith's best friend"). Em Perspectives on technology há um longo diálogo com Schumpeter, por meio de uma especial preocupação para lidar com a sua contribuição (ROSENBERG, 1976, p. 66-68). Há aqui, portanto, uma contribuição notável de Rosenberg: sua incursão na história do pensamento econômico enriquece a emergente elaboração evolucionária com um padrão de interpretação que posiciona a nova abordagem como herdeira dos clássicos da economia, ao demonstrar como para eles a tecnologia tem papel central na dinâmica econômica.

Além de trazer a história da economia e do pensamento econômico para a raiz da elaboração evolucionária, do ponto de vista crítico, dois capítulos de Perspectives on technology são sistematizaçóes de problemas da teoria econômica dominante no pós-guerra em relação ao progresso tecnológico: o capítulo 4 (Problems in the economist's conceptualization of technological innovation) e o capítulo 5 (Neglected dimensions in the analysis of economic change). Essas críticas serão importantes para a construção da elaboração neo-schumpeteriana como uma alternativa ao pensamento econômico mainstream.

Entre tantas ideias importantes, é necessário destacar que há ao menos três contribuiçôes teóricas decisivas de Rosenberg em Perspectives on technology.

A primeira está relacionada com a "orientação do progresso tecnológico" e com "mecanismos de focalização". ${ }^{5}$ No capítulo 6, intitulado "The direction of technological change: inducement mechanisms and focusing devices" (originalmente publicado em 1969), Rosenberg (1976, p. 110) sistematiza exemplos históricos para compreender a existência de "particular sequence and timing of innovative activity”, pois as explicações derivadas apenas de respostas a mudanças de preços de fatores seriam incompletas. Os exemplos históricos demonstram a existência de uma lógica específica que estabeleceria "sequências compulsivas" na busca de solução de problemas: "complex technologies create internal compulsions and pressures which, in turn, initiate exploratory activities in particular directions" (p. 111). A indústria automobilística apresenta um exemplo básico: a necessidade de freios melhores para lidar com motores mais potentes. Rosenberg (1976) utiliza aqui a terminologia de Hirschman: "backward linkages" seriam poderosos indutores do

5 Essa primeira contribuiçăo foi reproduzida na Revista Brasileira de Inovação, v. 11, n. 1, 2012, na seção Ideias fundadoras, com apresentação de Victor Pelaez. 
desenvolvimento da indústria de máquinas no mundo ocidental e, por sua vez, essa indústria foi decisiva no conjunto da industrialização (p. 112). Como mecanismos de focalização, Rosenberg (1976) descreve "desequilíbrios tecnológicos" (p. 112 117), "resistência de trabalhadores à disciplina" (exemplo retirado de Marx em $O$ Capital) (p. 117-120), "ruptura de fontes de recursos" (p. 121-122), "legislação" (p. 122-123) e outros fatores, incluindo "acidentes e outros desastres" (p. 123-124). ${ }^{6} \mathrm{~A}$ imprevisibilidade e a interdependência estão fortemente presentes nesses processos sequenciais de solução de problemas. Essa elaboração será posteriormente utilizada por Nelson e Winter (1977) na definição de trajetórias naturais - referências ao capítulo 6 e a Rosenberg (1972) - e por Dosi (1982) na formulação sobre trajetórias e paradigmas tecnológicos.

As duas outras contribuiçōes teóricas decisivas relacionam-se a uma discussão crítica com a herança schumpeteriana. Leitor e crítico de Schumpeter, Rosenberg adota o esquema simples sugerido por aquele autor tanto na Teoria do desenvolvimento econômico como no Business cycles (invenção-inovação-imitação) como ponto de partida para demonstrar como o processo é mais complexo, mais multifacetado e com mais estágios e atores do que inicialmente sugerido. Sinteticamente, Rosenberg (1976) avança por territórios não explorados por Schumpeter.

A segunda contribuição teórica decisiva está antes da inovação no esquema de Schumpeter: Rosenberg (1976) investiga até onde a atividade de invenção pode ser endogeneizada, até onde a atividade inventiva responde a fatores econômicos. Rosenberg $(1976$, p. 67, 73) questiona a completa exogeneidade da invenção. Em diálogo com Marx, um artigo discute o papel econômico da ciência e as complexas interaçôes entre ciência e tecnologia (p. 126-138). Em diálogo com Schmookler, outro artigo questiona a completa endogeneização do progresso tecnológico. Desses diálogos, o autor conclui que quando os limites do conhecimento científico são levados em conta o progresso tecnológico encontra limites temporariamente intransponíveis (ROSENBERG, 1976, p. 260-279). Essa elaboração prepara uma contribuição clássica, mas posterior, de Rosenberg (1981): o texto "How exogenous is science?”

A terceira contribuição teórica decisiva está depois da inovação no esquema de Schumpeter: Rosenberg questiona a subestimação da necessidade de criatividade nas

6 Nesses dois últimos tópicos, Rosenberg (1976) faz referência à construção da "tubular railroad bridge across the Menai Straits in 1840”, que teve de lidar com restrições impostas pela Marinha britânica (p. 122) e com uma explosão de um cilindro hidráulico (p. 124). Essas referências indicam um interesse do autor nessa construção, que levou a uma pesquisa e a um livro: The Britannia Bridge (ROSENBERG; VINCENTI, 1978).

7 Essa segunda contribuição decisiva - "Quão exógena é a ciência?" - também foi reproduzida pela Revista Brasileira de Inovação, v. 5, n. 2, 2006.com apresentação de Tamás Szmrecsányi. 
atividades de imitação - ou da "mera imitação", como escreve o autor da Teoria do desenvolvimento Econômico. Rosenberg (1976, p. 75) descreve o processo de difusão como "typically dependent upon a stream of improvements in performance characteristics of an innovation, its progressive modification and adaptation to suit the specialized requirements of various submarkets, and the availability and introduction of other complementary inputs which decisively affect the economic usefulness of an original innovation”. Essas observaçōes apoiam-se no capítulo 11 (Factors affecting the diffusion of technology), que trata da importância das atividades de inovação incremental para o processo de difusão da inovação: a difusão é "continuidade da atividade inventiva" (p. 191), há um processo de "melhorias nas invenções depois de sua primeira introdução" (p. 195), há o "desenvolvimento de habilidades técnicas entre usuários" (p. 197), há importantes "complementariedades" (p. 201) e inclusive "melhoras em tecnologias 'velhas"' (p. 202). Todos esses elementos contribuem para definir a velocidade da difusão da nova tecnologia - tema que reaparece em elaboraçôes posteriores de Rosenberg. O contexto institucional é avaliado no encerramento do capítulo, no tópico intitulado "Diffusion and its institutional context", no qual a discussão sobre a natureza das instituições antecipa uma preocupação geral da abordagem neoschumpeteriana com o tema, que terminará como uma das fontes da elaboração sobre sistemas de inovação nos anos 1980.

\section{Para além de Schumpeter: ciência endógena e inovação incremental}

Perspectives on technology apresenta a contribuição fundadora de Rosenberg para a emergente abordagem evolucionária, mas também indica uma agenda de pesquisa que será trabalhada a seguir.

Destacam-se nessa agenda os trabalhos que prosseguem na linha de detalhamento do esquema invenção-inovação-imitação. Ou seja, como inovações tecnológicas são geradas, testadas e difundidas.

Em relação ao estágio invenção-inovação, ao contrário do esquema de Schumpeter, não existe um estoque de invenções disponíveis para o inovador. A literatura sobre inovação "simply assumes the existence of a profitable invention and then goes on to try to account for the lag in its adoption" (ROSENBERG, 1976, p. 73). Schmookler, por sua vez, incorreu no problema oposto, ao concluir "that we can treat invention just like any other economic activity" (ROSENBERG, 1976, p. 263). O autor, crítico desses dois extremos, constrói uma elaboração sobre o desenvolvimento de invenções e suas relaçôes com a ciência. Por um lado, "economic 
forces and motives have inevitably played a major role in shaping the direction of scientific progress". Por outro lado, essas forças "have not acted within a vacuum, but within the changing limits and constraints of a body of scientific knowledge growing at uneven rates among its component subdisciplines" (ROSENBERG, 1976, p. 270). Essa lógica será mais desenvolvida no texto How exogenous is science?, em que se propõe a "to identify significant chains of causation running from economic life to science as well as from science to economic life" (ROSENBERG, 1982, p. 141). O ponto central do texto é "that technological concerns shape the scientific enterprise in various ways" (ROSENBERG, 1982, p. 141).

O primeiro passo é identificar que a influência de preocupações tecnológicas sobre o crescimento do conhecimento científico tem sido reconhecida há muito tempo. Torricelli, Carnot, Joule e Pasteur seriam exemplos de como "scientific knowledge of a wide generality grew out of a particular problem in a narrow context" (ROSENBERG, 1982, p. 143). A tecnologia deve ser vista como um corpo de conhecimentos que antecedem o conhecimento científico: altos-fornos e aviōes são exemplos de tecnologias que funcionavam sem respectivas teorias da combustão ou turbulência, demonstrando que é normal a experiência e o conhecimento tecnológico precederem o conhecimento científico. Assim, "technology has served as an enourmous repository of empirical knowledge to be scrutinized and evaluated by the scientist" (ROSENBERG, 1982, p. 144). Essa é uma sequência menos comum em indústrias fundadas na pesquisa científica - eletricidade, por exemplo -, mas mesmo aqui a experiência prática com nova tecnologia precede o conhecimento científico: um exemplo é a ciência do crescimento de cristais, que se desenvolve a partir da análise de curtos-circuitos em equipamento elétrico (p. 144-145). A relação entre o funcionamento do transistor e a física do estado sólido é o exemplo final nesse tópico (p. 145).

Outros canais por onde a tecnologia orienta o progresso da ciência são: "natural trajectory of certain technological improvements identifies and defines the limits of further improvements, which in turn, focuses subsequent scientific research" (ROSENBERG, 1982, p. 147) - os exemplos são a turbina a jato e ligação telefônica de longa distância -; mudanças no ambiente tecnológico que abrem espaço para a pesquisa básica - um exemplo aqui é o crescimento do ramo da ótica na física e o surgimento das fibras óticas e do laser (p. 154); rupturas tecnológicas que direcionam pesquisa científica - o primeiro transistor, o primeiro avião, o primeiro artefato nuclear sinalizam o início de uma série de desenvolvimentos, requerem um conjunto de inovações complementares e tecnologias auxiliares, tudo isso traduzido 
em novos incentivos econômicos para certas linhas de pesquisa; e instrumentos de observação, medida e teste - do microscópio ao computador, esses instrumentos mudam a qualidade e as possibilidades de pesquisas e são cada vez mais caros.

$\mathrm{Na}$ conclusão deste capítulo Rosenberg (1982, p. 159) afirma que "powerful economic impulses are shaping, directing and constraining the scientific enterprise": a ciência é cara e pode ser dirigida para onde atrairá retornos econômicos maiores. $\mathrm{O}$ processo de industrialização ao longo do século XX inevitavelmente transforma a ciência em uma atividade mais endógena, aumentando sua dependência em relação à tecnologia. Assim, Rosenberg (1982, p. 159) sugere "that a promising model for understanding scientific advances is one that combines the 'logic' of scientific progress with a consideration of costs and rewards that flow from daily life and are linked to science through technology".

Em relação ao estágio inovação-imitação, Rosenberg aperfeiçoa a compreensão da já seminal contribuição sobre o papel das inovaçōes incrementais para a difusão de inovaçôes. Em um artigo publicado originalmente em 1979 ("Technological interdependence in the American economy"), Rosenberg avalia que o interesse recente na difusão de tecnologias poderia ser interpretado como uma correção a uma visão heroica da invenção. Paradoxalmente, esse texto, em que o autor trata com detalhes da importância das pequenas melhorias incrementais para o conjunto da difusão, também discute um conjunto bem específico de invençôes-inovações de grande impacto: revolução industrial, eletrificação, motor de combustão interna, plásticos, eletrônica e computadores (ROSENBERG, 1982, p. 59).

O raciocínio de Rosenberg (1982, p. 56) se desenvolve discutindo que "inventions hardly even function in isolation”, como uma introdução à questão da complementariedade tecnológica na difusão das inovações. A dependência mútua de várias tecnologias é destacada, com as complexas relações de interdependência e interação que muitas vezes são de difícil percepção. É apresentada a abrangência do impacto das inovações que reduzem o custo de geração de energia ou o custo do transporte sobre uma cadeia de setores e regiōes (ROSENBERG, 1982, p. 57-58). A importância dessas complementariedades ressalta que "the social pay off of an innovation can rarely be identified in isolation" (p. 58).

Esse ponto de seu raciocínio leva Rosenberg (1982, p. 58-59) a discutir tecnologias especiais e de alto impacto na economia: "the growing productivity of industrial economies is the complex outcome of large number of interlocking, mutually reinforcing technologies, the individual components of which are of very limited economic consequences by themselves. The smallest unit of observation, therefore, 
is seldom a single innovation, but more typically, an interrelated clustering of innovations". Essa seria a forma de interpretar eventos como a revolução industrial: "interactions of a few basic technologies that provided the essential foundation for other technological changes in a series of ever-widening concentric circles, at the heart of which were a few major innovations in steam power, metallurgy (primarily iron), and the large-scale utilization of mineral fuels" (ROSENBERG, 1982, p. 59).

Outros exemplos desse tipo de clustering seriam encontrados em torno da eletrificação, dos plásticos, da eletrônica e de computadores. Segundo Rosenberg (1982, p. 59), "in each case a central innovation, or a small number of innovations, provided the basis around which a larger number of further cumulative improvements and complementary innovations were eventually positioned". Esse texto se completa com uma discussão do impacto cumulativo de pequenos melhoramentos e das relações interindustriais.

Nesse ponto da elaboração teórica de Rosenberg é possível sugerir a existência de um conjunto de evidências e reflexôes sobre revoluçôes tecnológicas - desde o insight tecnológico ou do ponto de partida científico, passando pela invenção e sua custosa e muitas vezes lenta transformação em inovação, até toda a complexa rede de atores e setores industriais e econômicos que interagem em sua difusão por meio de uma longa sequência de inovaçôes incrementais e pequenos melhoramentos.

\section{0 ceticismo quanto às ondas longas}

A acumulação de conhecimento sobre as revoluções tecnológicas é o ponto de partida de Rosenberg para a sua contribuição crítica no debate sobre as ondas longas do desenvolvimento capitalista.

$\mathrm{Na}$ emergente elaboração evolucionária há uma incursão inicial de Freeman (1977) sobre os ciclos de Kondratiev, seguida de um número especial da revista Futures (1981) editado por Freeman, ${ }^{8}$ posteriormente transformado em um livro (FREEMAN, 1983). Há ainda a publicação de uma obra coletiva sobre esse tema: Unemployment and technical innovation (FREEMAN; SOETE; CLARK, 1982).

Por um lado, as elaboraçôes de Rosenberg são utilizadas nesses trabalhos sua pesquisa histórica alimenta essa linha de elaboração. Freeman, Soete e Clark (1982, p. 64), discutindo "clusters of innovations and the diffusion of innovations", referem-se à trajetória natural das tecnologias, apoiando-se em Rosenberg (1976),

8 Disponível em: <http://www.sciencedirect.com/science/journal/00163287/13/4>. 
Nelson e Winter (1977) e Dosi (1982). . Mais adiante, os autores afirmam que "we agree wholeheartedly with Rosenberg's point (1976, Chapter 11) that the diffusion process cannot be viewed as one simple replication and carbon-copy imitation, but frequently involves a string of further innovations - small and large" (FREEMAN; SOETE; CLARK, 1982, p. 65).

Por outro lado, Rosenberg e Frischtak (1983) participam com um texto sintético - resumo de artigo publicado posteriormente no Cambridge Journal of Economics-, no qual apresentam seu ceticismo sobre as ondas longas do desenvolvimento capitalista:

No one who has examined the dynamics of capitalist economies over long historical periods can doubt that they experience significant long-term variations in their aggregate performance. The question is whether these long-term variations are more than the outcome of a summation of random events, and further, whether they exhibit recurrent temporal regularities that are sufficiently well-behaved to call them "long waves" (ROSENBERG; FRISCHTAK, 1983, p. 146).

Os autores sistematizam a crítica às elaborações de Kondratiev, Freeman, Mandel, Rostow e Schumpeter de uma forma que pode ser interpretada como um conjunto de questôes para um programa de pesquisa sobre o tema.

São quatro os desafios lançados à elaboração sobre ondas longas, questôes que requerem elaboração e respostas:

- causalidade: "it is essential to stress that an expoent of the view that technological change is at the root of the long cycle needs to demonstrate a) that changes in the rate of innovation govern changes in the rate of new investment and b) that the combined impact of innovation clusters takes the form of fluctuations in the aggregate output or employment" (ROSENBERG; FRISCHTAK, 1983, p. 147); ${ }^{10}$

- “timing": Rosenberg e Frischtak (1983, p. 147-148) listam tópicos que "may be expected to determine the length of the time period required for the introduction of new technologies and the realization of their full impact upon aggregate output": 1) "new inventions are typically very primitive at the

9 Como mencionado na seção 2, tanto Nelson e Winter (1977) quanto Dosi (1982) citam Rosenberg (1976). Uma versão deste texto de Dosi aparece no livro editado por Freeman (1983), como o seu capítulo 7.

10 Rosenberg e Frischtak (1983) não tiveram acesso a textos de Kondratiev publicados mais recentemente. Esses novos textos indicam que Kondratiev teria antecipado Schumpeter na colocação das inovaçōes tecnológicas entre os fatores causais dos movimentos de longo prazo na economia. Para Kondratiev (1926, p. 49), "the dynamics of long waves is induced by random attendant conditions and events, such as 1- changes in techonolgy, 2-wars and revolutions, 3- the involvement of new territories in the orbit of the world economy, 4- fluctuations in gold mining". 
time of birth"; 2) "adoption and diffusion rates are also powerfully shaped by expectation patterns"; 3) "the adoption of a new technology is critically dependent upon the availability of complementary inputs or, in some cases, upon an entire supporting infrastructure"; 4) em relação a diferentes inovaçōes, os autores levantam a questão sobre como elas poderiam se combinar: há espaçamento ou superposição entre essas diferentes inovações?

- repercussões econômicas: o impacto das inovações vai além delas próprias, envolvendo "backward linkages" e "forward linkages" (ROSENBERG; FRISCHTAK, 1983, p. 149-150), uma discussão dos impactos interindustriais: "the interindustry flow of new materials, components and equipment may generate widespread product improvement and cost reduction throughout the economy. This has clearly been the case in the past among a small group of producer goods industries - machine tools, chemicals, electrical and electronic equipment" (ROSENBERG; FRISCHTAK, 1983, p. 150). Como no máximo uma "untested hypothesis", os autores consideram que "it is certainly conceivable that innovations, depending upon their location, generate long cycles through such interindustry flows and their macroeconomic effects" (ROSENBERG; FRISCHTAK, 1983, p. 150).

- recorrência: a necessidade de demonstrar seu "cyclical or recurrent character".

A conclusão do texto é enfática em relação à inexistência de um modelo adequado que desse conta dessas quatro questôes - "until such model is developed, the assessment of its historical validity remains unresolved" (ROSENBERG; FRISCHTAK, 1983, p. 151).

\section{Instituições, mudanças institucionais e trajetórias de tecnologias}

Um período intermediário na elaboração de Rosenberg - entre a discussão crítica sobre as ondas longas (em 1983) e o primeiro texto publicado com um estudo voltado ao tema das GPTs (1998a) - é definido pela publicação de dois livros que articulam a visão sobre trajetórias tecnológicas e revoluçōes tecnológicas com processos históricos mais gerais e com o papel de instituições e suas transformaçōes: o primeiro sobre o Ocidente (ROSENBERG; BIRDZEL, 1986); ${ }^{11}$ e o segundo sobre os Estados Unidos (MOWERY; ROSENBERG, 1989). Essas obras indicam como

11 Rosenberg (1992) é um artigo que sumariza as ideias desse livro. 
Rosenberg participou do esforço coletivo relacionado ao então emergente conceito de sistema nacional de inovação. Rosenberg e Birdzel (1986) organizam um livro em torno de instituiçóes, mudança institucional e o papel da experimentação na história do capitalismo. O papel de mudanças institucionais fica evidente no capítulo 4 (The evolution of institutions favorable to commerce), que lista sistemas legais, inovações financeiras, como a letra de câmbio e seguro, a tributação, associações econômicas não familiares, técnicas contábeis, sistema moral e a fragmentação política europeia. O papel da mudança nas formas de organização das empresas é tratado no capítulo 6 (Diversity of organization: the corporation), que sistematiza a transição das sociedades limitadas para as sociedades anônimas, passando pelas sociedades por ações. Essas alterações estão vinculadas a mudanças discutidas no capítulo 7 (Technology, trusts and marketable stock), com ênfase para o desenvolvimento e a mudança na natureza das bolsas de valores, que estariam associadas à segunda revolução industrial (ROSENBERG; BIRDZEL, 1986, p. 238). O capítulo 8 sistematiza elaborações sobre o papel da ciência (The link between science and wealth), apresentando elementos sobre a diversidade das formas institucionais, tanto pelo lado da ciência básica como pelo lado da pesquisa industrial. Finalmente, no capítulo 9 (Diversity of enterprise) é discutido o papel específico - e mutante - de grandes e pequenas empresas.

Essa grande sistematização do papel da tecnologia na economia do Ocidente é seguida por um livro, escrito com D. Mowery em 1989, voltado para os Estados Unidos (Technology and the pursuit of economic growth). A estrutura institucional da ciência e da tecnologia nos Estados Unidos é o tema, em um trabalho articulado em torno da mudança estrutural estabelecida durante a Segunda Guerra Mundial. Três estruturas institucionais distintas são descritas, correspondendo a 1860-1940 (Parte II), a 1940-1987 (Parte III) e uma nova estrutura que se delineava a partir dos anos 1980 (Parte IV). Talvez como um exemplo de como as mudanças institucionais contribuem para moldar trajetórias tecnológicas e orientar novas direções do progresso tecnológico, o capítulo 7 (na Parte II) discute "The US commercial aircraft industry". Entre os temas tratados neste capítulo estão a contribuição da pesquisa científica de inspiração militar e de novas instituições como a Nasa.

Esses dois livros podem também ser interpretados como aperfeiçoamentos na elaboração de Rosenberg para dar conta de novos aspectos institucionais da endogeneização da ciência. A partir dessa sistematização, o autor posteriormente identificou a crescente endogeneização da ciência como um fenômeno do século XX (ROSENBERG, 2006). Essas reflexões estariam também relacionadas com 
uma elaboração sobre a natureza endógena das universidades dos Estados Unidos (ROSENBERG, 2000, capítulo 3)

Em 1993 é publicado o estudo comparado sobre sistemas de inovação editado por Richard Nelson. Rosenberg é coautor de Nelson em uma introdução que sistematiza um diálogo entre esses dois pioneiros da elaboração evolucionária. A introdução discute o entrelaçamento entre a ciência e a tecnologia - com a definição da ciência como "leader and follower" (NELSON; ROSENBERG, 1993, p. $6)^{12}$-, a descrição dos atores institucionais mais importantes -, firmas, laboratórios industriais de pesquisa, universidades e laboratórios governamentais, entre outros, com especial atenção para diferenças interindustriais -, comunidades tecnológicas, fronteiras e sistemas nacionais de inovação e as tensões entre sistemas nacionais e tecnologias transnacionais. Rosenberg ainda participa, juntamente com Mowery, no capítulo sobre os Estados Unidos - em grande parte apoiado no livro de 1989.

Em 1994 a Reseach Policy publicou um número especial em homenagem a Rosenberg, ${ }^{13}$ cujos artigos podem ser indicações de linhas representativas das contribuições do autor até então: três textos lidam com a ciência e a divisão de trabalho inovativo; um artigo aborda as inovações incrementais, outros dois artigos apresentam uma perspectiva mais geral (uma economia política da mudança tecnológica) - baseados no livro com Birdzel; e três artigos trazem estudos setoriais (ou sobre trajetórias tecnológicas selecionadas: química e aviação).

Trajetórias tecnológicas ligadas à tecnologia médica entram na agenda de pesquisa em Rosenberg, Gelijns e Dawkins (1995), forjando uma linha de investigação relativa a um setor particularmente baseado na ciência.

Em 1996, um novo texto clássico - "Uncertainty and technological change" - parece ser uma das primeiras oportunidades em que Rosenberg menciona o conceito de revoluções tecnológicas de forma mais sistemática. O autor explicita, schumpeterianamente, que a maior parte das tentativas de inovar fracassa, sendo que o tema do capítulo seria discutir "the inability to antecipate the future impact of successful innovations, even after their technical feasibility has been established" (ROSENBERG, 1996, p. 153). Para ilustrar isso, o autor seleciona exemplos relacionados às mais importantes inovações do século XX. Há uma longa lista de dilemas,

12 É também desse período um aperfeiçoamento da elaboração da relação entre ciência e tecnologia (KLINE; ROSENBERG, 1986). Certamente essa elaboração contribuiu para a formulação apresentada em conjunto com Nelson. Este texto de Kline e Rosenberg (1986) também foi reproduzido pela Revista Brasileira de Inovação, v. 14, n, 1, 2015, na seção Ideias fundadoras, com apresentação de Alessandro Maia Pinheiro.

13 Disponível em: <http://www.sciencedirect.com/science/journal/00487333/23/5>. 
novas incertezas que surgem à medida que problemas são resolvidos e novos desafios surgem. $\mathrm{O}$ raciocínio relativo a esta longa sequência de novos desafios e novas incertezas pode ser captado pela mudança no título do capítulo introduzida por Rosenberg (2010): "Improvement upon improvement: long after innovation". Os casos apresentados são do laser, das fibras óticas, do telefone, do rádio, do telefone sem fio, do computador e do transistor. Esses casos levam Rosenberg a concluir, em uma importante nota teórica: "indeed, the uncertainty associated with the eventual uses of the laser or the computer might, more appropriately, be characterized as 'ignorance' rather than as 'uncertainty" (ROSENBERG, 2010, p. 159), ignorância que tem um papel importante no processo de mudança técnica.

Dessas reflexōes, Rosenberg (2010, p. 162) indicou que "major new technological regimes take many years before they replace an established technology". Para ele, "a radical new technology such as a computer must necessarily have a long gestation period before its characteristics and opportunities are well understood and can be throughly exploited" (ROSENBERG, 2010, p. 163). Assim, "history strongly suggests that technological revolutions are not completed overnight" (ROSENBERG, 2010, p. 163).

\section{0 diálogo com a elaboração das GPTs: o historiador de revoluções tecnológicas}

Em 1998 Helpman editou o livro General purpose technologies. Para ele, "a drastic innovation qualifies as a GPT if it has the potential for pervasive use in a wide range of sectors in ways that drastically change their mode of operation" (HELPMAN, 1998, p. 3). A partir de um capítulo do livro, Helpman (1998, p. 5) menciona uma caracterização baseada em quatro características principais: "(1) much scope for improvement initially, (2) many varied uses, (3) applicabilty across large parts of the economy, (4) strong complementarities with other technologies".

Esse livro é importante, porque Rosenberg participa simultaneamente como autor do capítulo 7 e como referência bibliográfica em outros cinco capítulos. ${ }^{14} \mathrm{Na}$ introdução, Helpman atribui a Bresnahan e Trajtenberg (1995) a autoria do termo general purpose technologies (GPTs). Uma leitura desse texto pioneiro de GPTs fornece alguns elementos para a identificação da influência de Rosenberg sobre a origem deste conceito. Em primeiro lugar, Bresnahan e Trajtenberg (1995) agradecem os

14 Uma indicação de um diálogo com a abordagem das ondas longas é a referência a textos de Freeman (às vezes com Perez) em quatro capítulos de Helpman (1998). 
comentários de Rosenberg a versôes anteriores do texto. Em segundo lugar, eles citam o livro Inside the black box diversas vezes, como apoio à ideia das GPTs. Em terceiro lugar, em uma nota de rodapé, os autores esclarecem que "[i]n defining innovational complementarities and understanding their role, we were strongly influenced by Rosenberg's insightful 1979 essay, 'Technological interdependence in the American economy"' (BRESNAHAN; TRAJTENBERG, p. 84). Em quarto lugar, eles indicam ao leitor uma versão maior deste texto (publicado em 1992 como Working Paper da National Bureau of Economic Research), que tem uma seção histórica com outras referências a Rosenberg.

Em uma comunicação por e-mail, Roberto Mazzoleni - orientado por Rosenberg na Stanford University - lembra-se da passagem de Manuel Trajtenberg por Stanford no início dos anos 1990. Segundo Mazzoleni:

I would certainly agree that Nate was fairly enthusiastic about the concept of GPT. If I am correct, the first paper using that term was written by Manuel Trajtenberg and Tim Bresnahan. At that time, Manuel was visiting Stanford, and I am pretty sure that the interactions between the two of them and Nate focused on the similarities between their concept of GPT and Nate's earlier concept of technological convergence, which was central to Nate's discussion of the role of machine tools in nineteenth century industrial progress. Since I was working on numerical control for machine tools, I was exposed to a bit of that conversation. Later on, a larger project got underway that led to the GPT book edited by Elhanan Helpman. ${ }^{15}$

O próprio Rosenberg, posteriormente, na introdução do capítulo 5 do livro Schumpeter and the endogeneity of technology, em 2000, em que é republicado o capítulo do livro editado por Helpman em 1998, comenta favoravelmente os esforços dessa elaboração emergente:

In the first half of the 1990s economists were beginning to explore the notion that some technological innovations were of special significance because they provided building blocks upon which numerous other innovations were eventually constructed. That is, some technologies can be regarded as general purpose technologies (GPTs) because they provided technological capabilities that could be utilized in a large number of "application sectors". Steam engines, machine tools, electricity, transistors, and computers all fall in this category (ROSENBERG, 2000, p. 80). 
Ao mesmo tempo, ele apresenta uma reconsideração da sua obra à luz dessa abordagem: "This way of thinking seemed eminently sensible and specially illuminating to someone who has long had a special interest in the history of technologies, and who had in fact written a paper many years ago that exploited the relationship between the introduction of a new class of machines - machine tools - and a wide range of subsequent applications" (ROSENBERG, 2000, p. 80). Em uma nota de rodapé, o autor indica aos leitores dois artigos: "Technological change in the machine tool industry", escrito em 1963, e "Technological interdependence in the American economy", produzido em 1979. Articulando esses textos com a literatura de GPTs, Rosenberg (2000, p. 116) escreve: "This second paper, drawing in part on the earlier one, pays special attention to two issues of special significance in the latter GPT literature: the role of complementarities among technologies and the importance of interindustry complementarities".

A partir de 1998, Rosenberg elabora ao menos três textos utilizando o conceito de GPT, que abordam a engenharia química (ROSENBERG, 1998a), a eletricidade (ROSENBERG, 1998b) e máquina a vapor (ROSENBERG; TRAJTENBERG, 2004).

É também de 1998 outro livro escrito em conjunto com Mowery, Paths of innovation, no qual são discutidas quatro trajetórias tecnológicas: motor a combustão (capítulo 3); química (capítulo 4); energia elétrica (capítulo 5); e a "revolução da eletrônica" (capítulo 6). Esse livro apresenta uma visão sobre a importância da mudança institucional para que o papel das relações complexas entre ciência e tecnologia tenha lugar ao longo das trajetórias das revoluções tecnológicas - o capítulo 1 de Paths of Innovation lida com mudanças institucionais no sistema de inovação dos Estados Unidos. Essa introdução é necessária para captar a diversidade das contribuiçõos da ciência ao longo das trajetórias tecnológicas apresentadas na sequência. Ao final do livro, no capítulo 7, há uma discussão sobre "the changing economic role of science" e sobre como a ciência tem se tornado cada vez mais endógena ao sistema (MOWERY; ROSENBERG, 1998). ${ }^{16}$

Dosi e Nelson (2010, p. 66) identificam a posição específica das GPTs em relação às elaborações prévias sobre trajetórias tecnológicas:

16 A partir de 1995, o conceito de GPTs adquire vida própria, passando a ser referência para discussōes em diversas áreas da economia. Dois exemplos correspondem à presença de capítulos relativos a GPTs em handbooks de áreas como crescimento econômico (JOVANIC; ROUSSEAU, 2005) e economia da inovação (BRESNAHAN, 2010). Nesse último caso, trata-se de um livro coeditado por Rosenberg. 
[h]ere as well as in Dosi (1982), we use the notion of paradigm in a microtechnological sense: for example, the semiconductor paradigm, the internal combustion engine paradigm, etc. This is distinct from the more "macro" notion of "techno-economic paradigm" used by Perez $(1985,2010)$ and Freeman and Perez (1988) which is a constellation of paradigms in our narrow sense: for example, the electricity techno-economic paradigm, ICTs, etc. The latter broader notion overlaps with the idea of 'general purpose technologies' from Bresnahan and Trajtenberg (1995).

O conceito de GPTs tem aberto uma possibilidade de diálogo entre diversas correntes da economia, talvez um caso singular de diálogo intradisciplinar - textos como Atkeson e Keohe (2007) demonstrariam isso, ao conter, entre as referências, tanto o livro de Helpman (1998) como um artigo de Rosenberg ("On technological expectations”, publicado em Inside the black box). Esse diálogo pode ser mais explorado em outra ocasião, mas deve-se insistir na importância dessa contribuição de Rosenberg para a disciplina da economia em geral: o historiador das revoluçōes tecnológicas, com as suas "historical reconnaissance missions", é também o teórico das suas múltiplas determinações e contribuiu para sistematizações que foram utilizadas pelo esforço de formalização em torno das GPTs.

Em termos metodológicos, há aqui um interessante caso de relacionamento entre uma teoria apreciativa e uma teoria formal. Nelson e Winter (1982) avaliam que uma elaboração fértil na teoria econômica pressupõe uma relação entre teorização apreciativa e sua formalização. Assim, Rosenberg é o elaborador da teoria apreciativa que alimenta as GPTs como esforço de formalização. Como foi apresentado nesta seção, há um diálogo explícito entre Rosenberg e os pioneiros da formalização das GPTs. A teoria formal derivada das GPTs, por sua vez, contribui para colocar o tema das revoluções tecnológicas em discussão para além do campo dos diretamente interessados na economia da inovação.

\section{Rosenberg, GPTs e uma agenda de pesquisa}

O legado de Rosenberg vai muito além da sua contribuição para a emergência das GPTs, como o conjunto de sua obra demonstra e como indicam tanto o número especial da Research Policy em 1994 como o seminário da Industrial and Corporate Change em 2016. ${ }^{17}$

17 Disponível em: <http://businessinnovation.berkeley.edu/intellectual-capital/icc-conference>. 
Este artigo, porém, explora a participação de Rosenberg na emergência e difusão do conceito de GPT, porque aqui pode estar o elemento paradoxal sugerido na introdução: o desdobramento das pesquisas relativas às GPTs recoloca questôes sobre a dinâmica de longo prazo na economia capitalista e retoma temas abertos por Kondratiev.

Dosi e Nelson (2010) listam a contribuição das GPTs ao lado das pesquisas de Freeman, Perez e Louçã. Para eles,

[a] full evolutionary account of economic growth would also take into account that the historical time path of growth tends to be punctuated by "eras" characterized by the development and diffusion of specific constellations of "general-purpose" technologies (Bresnahan and Trajtenberg, 1995; Rosenberg and Trajtenberg, 2004), that is broad techno-economic paradigms in the sense of Perez (1985), Freeman and Perez (1988), and Freeman and Louçã (2001). During a particular economic era, much of the economic growth is accounted for by innovation and productivity growth in the industries that produce the goods that directly incorporate the driving technological paradigms and also in the downstream industries that are able to use these goods as inputs (historically, this was the case of steam power, later electricity and the internal combustion engine, and today it is the case of ICT technologies) (DOSI; NELSON, 2010, p. 113).

Não é casual que, em texto mais recente, Bresnahan (2010) discuta em uma seção o tema "aggregate growth waves", com explícitas referências a "ondas longas" que teriam tido uma retomada de interesse após o debate em torno do "paradoxo da produtividade" comentado por Solow - e com uma menção ao próprio Kondratiev. $\mathrm{O}$ autor refere-se a um vasto conjunto de trabalhos que busca investigar a relação entre GPTs e longos ciclos, uma sequência iniciada por Helpman e Trajtenberg (1998), que "have investigated the possibility of long macroeconomic cycles associated with the invention and diffusion of GPTs" (BRESNAHAN, 2010, p. 786). ${ }^{18}$

Essa elaboração, enfim, enriquece uma agenda de pesquisa desafiante, reconhecida por Dosi e Nelson (2010, p. 133): “[g]ranted that, the relationship between techno-economic paradigms (and even more so individual general-purpose technologies thereof), on the one hand, and growth patterns, on the other, continues to be a challenging area of investigation".

18 Bresnahan (2010, p. 786) indica ao leitor um texto de Jovanovic e Rousseau (2005) - um capítulo do Handbook of Economic Growth, editado por Aghion e Durlauf - como uma sistematizaçāo de estudos empíricos nessa linha. 
Essa área de investigação é promissora por muitas razões. Em primeiro lugar, conforme comentado na seção 4, há escritos de Kondratiev que não estavam disponíveis para os debates dos anos 1970 e 1980 (MAKASHEVA et al., 1998). Essa literatura apresenta a elaboração de Kondratiev como mais sofisticada, nuançada e apoiada na literatura econômica de seu tempo do que sugeriam os seus textos mais conhecidos até então. Seria extremamente valioso tratar essas novas publicações de Kondratiev a partir das liçóes legadas por Rosenberg como historiador do pensamento econômico.

Em segundo lugar, existe uma questão metodológica sobre a relação entre teoria apreciativa e teoria formal. No diálogo entre a teoria apreciativa elaborada por Rosenberg e a formalização implementada por Bresnahan, Trajtenberg, Helpman e outros, há uma questão importante relativa à natureza da formalização: a adequação do instrumental da modelagem utilizado nessas tentativas. Pode-se conjecturar que o instrumental mais adequado para formalizar a teoria apreciativa desenvolvida por Rosenberg seria o conjunto de ferramentas disponibilizadas pelas teorias da complexidade. A modelagem derivada das teorias da complexidade permite incorporar as múltiplas interaçóes, os feedbacks positivos, os circuitos virtuosos, a incerteza e outros fenômenos relacionados identificados pela teoria apreciativa desenvolvida por Rosenberg.

Em terceiro lugar, um diálogo entre uma sugestão recente de Perez (2010, p. 190) e o conjunto da elaboração de Rosenberg seria fértil, constituindo um ponto importante para enfatizar o elemento paradoxal sugerido na introdução deste artigo. Perez (2010) sistematiza a dinâmica das ondas longas de uma forma esquemática, mas original, ao buscar identificar as inovaçōes que representariam os big bangs deflagradores das revoluçôes tecnológicas. Ler esse esquema proposto por Carlota Perez retendo as lições de Rosenberg estimula a visualização de um quadro mais geral, em que há superposição dos efeitos de diversos big bangs ao longo do tempo, de efeitos de diferentes revoluções tecnológicas em diversos momentos. Rosenberg sistematizou como desdobra-se ao longo do tempo a difusão das tecnologias centrais de cada revolução, com longos processos de inovaçóes incrementais e complementares. Essa abordagem abre espaço para essa outra investigação - como os desdobramentos de diferentes revoluçōes tecnológicas se combinam, se articulam e se entrelaçam. Essas combinações de consequências de revoluções tecnológicas distintas podem trazer novos elementos para a dinâmica econômica. Para esse debate específico, Rosenberg (1998a) ilustra essa superposição de duas trajetórias tecnológicas distintas ao estudar a combinação entre a engenharia química e o motor a combustão. Em primeiro 
lugar, a "invenção do automóvel" transformou a indústria de petróleo, que antes era um produto utilizado apenas para iluminação e lubrificação. Os esforços para extrair combustível do petróleo "provided much of the technological basis for a far larger group of industries - petrochemical industries - in the postwar years" (ROSENBERG, 1998a, p. 181). A explosão do consumo de gasolina nos Estados Unidos entre 1910 e 1930 forçou o desenvolvimento de novas tecnologias e capacitaçôes para atender a tal demanda, o que levou ao surgimento de "enormous unanticipated consequences": a atual indústria petroquímica (ROSENBERG, 1998a, p. 180), entre elas. A superposição dessas duas GPTs deve ter efeitos macroeconômicos não desprezíveis, sendo um exemplo de discussões que concretizam algumas das preocupaçôes críticas de Rosenberg em relação às ondas longas. A construção de modelos que incluam esse tipo de fenômeno tecnológico e econômico é um desafio importante.

Esses três pontos são apenas algumas sugestôes pontuais de uma área plena de desafios lançados por Rosenberg e sua obra. Há um enorme depósito de conhecimento teórico e de desafios críticos distribuídos na obra de Rosenberg, um legado riquíssimo e essencial para pesquisas que busquem ampliar a nossa compreensão da dinâmica tecnológica e de sua articulação com a economia e a sociedade.

\section{Referências bibliográficas}

ATKESON, A.; KEOHE, P. Modeling the transition to a new economy: lessons from two technological revolutions. American Economic Review, v. 97, n. 1, p. 64-88, 2007.

BRESNAHAN, T. General purpose technologies. In: HALL, B.; ROSENBERG, N. (Ed.). Handbook of the economics of innovation. Volume II. Amsterdam: North Holland, 2010. p. 761-791.

BRESNAHAN, T.; MALERBA, F. Industrial dynamics and the evolution of firms' and nations' competitive capabilities in the world computer industry. In: MOWERY, D.; NELSON, R. (Ed.). Sources of industrial leadership: studies in seven industries. Cambridge: Cambridge University Press, 1999.

BRESNAHAN, T.; TRAJTENBERG, M. General purpose technologies: 'engines of growth'? Cambridge, Mass: NBER, 1992 (Working paper, 4148).

- General purpose technologies: 'engines of growth'? Journal of Econometrics, v.

65, n. 1, p. 83-108, 1995. 
DAVID, P.; WRIGHT, G. General purpose technologies and surges in productivity: historical reflections on the future of the ICT Revolution. Oxford: University of Oxford, 1999 (Discussion papers in economic and social history, n. 31).

DOSI, G. Technical paradigms and technological trajectories: a suggested interpretation of the determinants of technical change. Research Policy, v. 2, n. 3, p. 147-62, 1982.

DOSI, G.; NELSON, R. Technical change and industrial dynamics as evolutionary processes. In: HALL, B.; ROSENBERG, N. (Ed.). Handbook of the economics of innovation. Volume I. Amsterdam: North Holland, 2010. p. 51-127.

FREEMAN, C. The economics of industrial innovation. Harmondsworth: Penguin, 1974. The Kondratiev long waves, technical change and unemployment. In: OECD. Structural determinants of employment and unemployment. Vol. 2. Paris: OECD, 1977. p. 181-196.

. (Ed.). Long waves in the world economy. London: Frances Pinter (Publishers) Ltd., 1983.

Critical survey: the economics of technical change. Cambridge Journal of Economics, v. 18, n. 5, p. 463-514, 1994.

The greening of technology and models of innovation. Technological Forecast and Social Change, v. 53, p. 27-39, 19996.

FREEMAN, C.; LOUÇÃ, F. As time goes by: from the industrial revolution to the information revolution. Oxford: Oxford University, 2001.

FREEMAN, C.; PEREZ, C. Structural crisis of adjustment: business cycles and investment behaviour. In: DOSI, G.; FREEMAN, C.; NELSON, R.; SILVERBERG, G.; SOETE, L. (Ed.). Technical change and economic theory. London: Pinter, 1988. p. 38-66.

FREEMAN, C.; SOETE, L. The economics of industrial innovation. London: Pinter, 1997. FREEMAN, C.; SOETE, L.; CLARK, J. Unemployment and technical innovation: a study of long waves and economic development. London: Frances Pinter, 1982.

HELPMAN, E. (Ed.). General purpose technologies. Stanford: Stanford University, 1998.

HELPMAN, E.; TRAJTENBERG, M. A time to sow and a time to reap: growth based on general purspose technologies. In: HELPMAN, E. (Ed.). General purpose technologies. Stanford: Stanford University. p. 55-83.

JOVANOVIC, B.; ROUSSEAU, P. General purpose technologies. Cambridge, Mass: NBER (Working paper, 11093). 
KLINE, S.; ROSENBERG, N. An overview of innovation. Revista Brasileira de Inovação, v. 14, n. 1, p. 9-48, [1986], 2015.

KONDRATIEV, N. D. Long cycles of economic conjuncture. In: MAKASHEVA, N.; SAMUELS, W.; BARNETT, V. (Ed.). The works of Nikolai D. Kondratiev. London: Pickering and Chato [1926], 1998, p. 25-60.

MAKASHEVA, N.; SAMUELS, W.; BARNETT, V. (Ed.). The works of Nikolai D. Kondratiev. London: Pickering and Chato, 1998.

MOWERY, D.; NELSON, R.; STEINMULLER, W. Introduction: in honor of Nathan Rosenberg. Research Policy, v. 23, n. 5, pp. iii-v, 1994.

MOWERY, D.; ROSENBERG, N. (1989) Technology and the pursuit of economic growth. Cambridge: Cambridge University Press.

. The US national innovation system. In: NELSON, R. (Ed.). National innovation systems: a comparative analysis. New York, Oxford: Oxford University, 1993. p. 29-75.

- Paths of innovation: technological change in 20th-century America. Cambridge:

Cambridge University Press, 1998.

NELSON, R. (Ed.). National innovation systems: a comparative analysis. New York, Oxford: Oxford University, 1993.

NELSON, R.; ROSENBERG, N. Technical innovation and national systems. In: NELSON, R. (Ed.). National innovation systems: a comparative analysis. New York, Oxford: Oxford University, 1993. p. 3-21.

NELSON, R.; WINTER, S. In search of useful theory of innovation. Research Policy, v. 6, n. 5, 1977.

An evolutionary theory of economic change. Cambridge, Mass; London: The Belknap Press of Harvard University Press, 1982.

PEREZ, C. Microelectronics, long waves and world structural change: new perspectives for developing countries. World Development, v. 13, n. 3, p. 441-63, 1985.

. Technological revolutions and techno-economic paradigms. Cambridge Journal of Economics, v. 34, n. 1, p. 185-202, 2010.

ROSENBERG, N. (Ed.). American system of manufactures: the report of the commitee on the machinery of the United States 1855 and the special reports of George Wallis and Joseph Whitworth 1854. Edited with an introduction by Nathan Rosenberg. Edinburgh: Edinburgh University Press, 1969. 
. Technology and American economic growth. Armonk: M. E. Sharpe, 1972.

. Karl Marx on the economic role of science. In: ROSENBERG, N. Perspectives on technology. Cambridge: Cambridge University Press, [1974a], 1976. p. 126-138.

Science, invention and economic growth. In: ROSENBERG, N. Perspectives on technology. Cambridge: Cambridge University Press [1974b], 1976. p. 260-279.

Problems in the economist's conceptualization of technological innovation. In: ROSENBERG, N. Perspectives on technology. Cambridge: Cambridge University Press, [1975], 1976. p. 61-84.

. Perspectives on technology. Cambridge: Cambridge University Press, 1976.

. The direction of technological change - inducement mechanisms and focusing devices. Revista Brasileira de Inovaçāo, v. 11, n. 1, p. 13-36, [1969], 2012.

Quão exógena é a ciência? Revista Brasileira de Inovação, v. 5, n. 2, p. 245-271, [1981], 2006.

Inside the black box: technology and economics. Cambridge: Cambridge University Press, 1982.

. Why do firms do basic research (with their money)? Research Policy, v. 19, p.165-174, 1990.

. Economic experiments. Industrial and Corporate Change, v. 1, n. 1, p. 181203, 1992.

The emergence of economic ideas: essays in the history of economics. Aldershot: Edward Elgar, 1994.

Uncertainty and technical change. In: LANDAU, R.; TAYLOR, T.; WRIGHT, G. The mosaic of economic growth. Stanford: Stanford University, 1996.

Chemical engineering as a general purpose technology. In: HELPMAN, E. General purpose technologies and economic growth. Cambridge, Mass./London: The MIT Press, 1998a. p. 167-192.

The role of electricity in industrial development. In: ROSENBERG, N. Studies on science and the innovation process - selected works by Nathan Rosenberg. New Jersey/ London: World Scientific, [1998b], 2010. p. 137-151.

. Schumpeter and the endogeneity of technology: some American perspectives.

London: Routledge, 2000. 
Studies on science and the innovation process - selected works by Nathan Rosenberg. New Jersey/London: World Scientific, 2010.

ROSENBERG, N.; BIRDZELL JR., L. E. How the west grew rich: the economic transformation of the industrial world. New York: Basic Books, 1986.

ROSENBERG, N.; FRISCHTAK, C. Long waves and economic growth: a critical appraisal. American Economic Review, v. 73, n. 2, p. 146-151, 1983.

ROSENBERG, N.; GELIJNS, A.; DAWKINS, H. Sources of medical technology: universities and industry (Medical innovation at the crossroads, v. 5). Washington: National Academy Press, 1995.

ROSENBERG, N.; TRAJTENBERG, M. A general purpose technology at work: the Corlis steam engine in the late-nineteenth-century United States. In: ROSENBERG, N. Studies on science and the innovation process - selected works by Nathan Rosenberg. New Jersey/ London: World Scientific, [2004], 2010. p. 97-136.

ROSENBERG, N.; VINCENTI, W. G. The Britannia Bridge: the generation and difusion of technological knowledge. Cambridge, Mass/London: The MIT Press, 1978.

SCHUMPETER, J. A teoria do desenvolvimento econômico. São Paulo: Nova Cultural, [1911], 1985.

. Business cycles: a theoretical, historical and statistical analysis of the capitalist process. Philadelphia: Porcupine, [1939], 1989.

. Capitalismo, socialismo e democracia. Rio de Janeiro: Zahar Editores, [1942], 1984. 